\title{
Path Relinking for Unconstrained Binary Quadratic Programming
}

\author{
Yang Wang ${ }^{\mathrm{a}}$, Zhipeng Lü ${ }^{\mathrm{b}}$, Fred Glover ${ }^{\mathrm{c}}$ Jin-Kao Hao ${ }^{\mathrm{a}, *}$ \\ ${ }^{a}$ LERIA, Université d'Angers, 2 Boulevard Lavoisier, 49045 Angers, France \\ ${ }^{\mathrm{b}}$ School of Computer Science and Technology, Huazhong University of Science and \\ Technology, 430074 Wuhan, China \\ ${ }^{\mathrm{c}}$ OptTek Systems, Inc., 2241 17th Street Boulder, CO 80302, USA
}

(Accepted to EJOR, 5 July 2012. DOI:

http://dx.doi.org/10.1016/j.ejor.2012.07.012)

\begin{abstract}
This paper presents two path relinking algorithms to solve the unconstrained binary quadratic programming (UBQP) problem. One is based on a greedy strategy to generate the relinking path from the initial solution to the guiding solution and the other operates in a random way. We show extensive computational results on five sets of benchmarks, including 31 large random UBQP instances and 103 structured instances derived from the MaxCut problem. Comparisons with several state-of-theart algorithms demonstrate the efficacy of our proposed algorithms in terms of both solution quality and computational efficiency. It is noteworthy that both algorithms are able to improve the previous best known results for almost 40 percent of the 103 MaxCut instances.

Keywords: UBQP, Path Relinking, Tabu Search, MaxCut
\end{abstract}

\section{Introduction}

The objective of the unconstrained binary quadratic programming (UBQP) problem is to maximize the function:

\footnotetext{
* Corresponding author.

Email addresses: yangw@info.univ-angers.fr (Yang Wang), zhipeng.lui@gmail.com, zhipeng.lv@hust.edu.cn (Zhipeng Lü), glover@opttek.com (Fred Glover), hao@info. univ-angers.fr (Jin-Kao Hao).
} 


$$
f(x)=x^{\prime} Q x=\sum_{i=1}^{n} \sum_{j=1}^{n} q_{i j} x_{i} x_{j}
$$

where $Q=\left(q_{i j}\right)$ is an $n$ by $n$ matrix of constants and $x$ is an $n$-vector of binary (zero-one) variables, i.e., $x_{i} \in\{0,1\}, i=1, \ldots, n$.

The formulation of UBQP can represent a wide range of important problems, including those from financial analysis [28], social psychology [20], computer aided design [25] and cellular radio channel allocation [9]. Moreover, a quite number of combinatorial optimization problems can be transformed into UBQP, such as graph coloring problem, maxcut problem, set packing problem, set partitioning problem, maximum clique problem, etc. Interested readers can refer to [23] for the general transformation procedures.

Given the interest of UBQP, many solution procedures have been reported in the literature during the past few decades. Exact methods based on branch and bound or branch and cut $[6,21,35]$ are quite useful to obtain optimal solutions to instances of limited sizes. To handle larger instances, a number of heurisric and metaheuristic methods have been developed, including local search [7], Simulated Annealing [4,22], Tabu Search [14,19,32,34,37,38], and Evolutionary and Memetic Algorithms [5,26,27,30,31].

Among the existing heuristics, tabu search (TS) based algorithms are the most successful ones. For example, the first adaptive memory tabu search algorithm for the UBQP [14] has been used to solve applications coming from a wide variety of settings. Also, several multi-start tabu search strategies have been explored in [32] and a sequel using an iterated tabu search algorithm has been investigated in [34], leading to very good results on large and challenging UBQP random instances. More recently, the diversification-driven tabu search method [19], a memetic algorithm [27] using embedded tabu search and a variable fixing tabu search method $[37,38]$ have proved to be especially effective for solving the most challenging UBQP instances.

Although numerous algorithms and approaches have been proposed for this well-known problem, we are not aware of any study on applying path relinking to the UBQP in the literature. Path relinking is a general search strategy closely associated with tabu search and its underlying ideas share a significant intersection with the tabu search perspective [15-17], with applications in a variety of contexts where it has proved to be very effective in solving difficult problems. In this paper, we follow the general scheme described in [17] and propose two path relinking algorithms for the UBQP. These two algorithms differ from each other mainly on the way of generating the path, one employing a greedy strategy and the other employing a random construction. In order to 
assess the performance of our path relinking algorithms, we provide computational results on five sets of random and structured benchmarks with a total of 134 test instances. These results indicate that our proposed algorithms yield highly competitive outcomes on the tested instances.

The remaining part of the paper is organized as follows. Section 2 briefly reviews some representative approaches for the UBQP. Section 3 describes the ingredients of our path relinking algorithms. Section 4 presents computational results and detailed comparisons with other state-of-the-art algorithms in the literature. Section 5 discusses the results obtained on two other well-known combinatorial problems. Concluding remarks are given in Section 6 .

\section{Previous Work}

This section reviews some representative heuristic approaches for the UBQP, including in particular those that are used as the reference methods for our experimental evaluation.

Glover et al. [14] introduced the first tabu search algorithm for the UBQP (AMTS). AMTS is based on the one-flip move and two types of memory structures to record recency and frequency information. Strategic oscillation is employed to alternate between constructive phases (progressively setting variables to 1) and destructive phases (progressively setting variables to 0), which are triggered by critical events, i.e., when the next move causes the objective function to decrease. The amplitude of the oscillation is adaptively controlled by a span parameter. Computational results for instances with up to 500 variables show AMTS outperforms the best exact and heuristic methods previously reported in the literature.

Katayama et Narihisa [22] designed a simulated annealing algorithm (SA) that is also based on the one-flip move and an incremental neighborhood evaluation technique. To enhance its search ability, the SA algorithm adopts multiple annealing processes starting from different temperatures. Tested on instances with variables ranging from 500 to 2500, the proposed SA heuristic shows very competitive performances, particularly for the largest instances.

Merz et Katayama [31] conducted a landscape analysis and observed that local optima of the UBQP instances are contained in a small fraction of the search space. Based on this, they designed a memetic algorithm (MA) in which a dedicated crossover operator is utilized to generate good starting solutions for a k-opt local search. The proposed approach is remarkably effective in solving a set of problems with up to 2500 variables. 
Palubeckis [32] presented several multistart tabu search strategies (MST) dedicated to the construction of the initial solution. An additional set of challenging random instances with up to 7000 variables were generated to evaluate the proposed MST algorithms. Subsequently, Palubeckis [34] developed an iterated tabu search algorithm (ITS) in which the perturbation mechanism operates on a specific set of variables. The experimental results indicated that the ITS consumes less computational effort to find the best solutions than several MSTS algorithms.

Glover et al. [19] presented a diversification-driven tabu search ( $\left.\mathrm{D}^{2} \mathrm{TS}\right)$ algorithm that alternates between a basic tabu search procedure and a memorybased perturbation strategy guided by a long-term memory. Despite its simplicity, computational results showed that $\mathrm{D}^{2} \mathrm{TS}$ is capable of matching or improving the previously reported results for the challenging instances introduced in $[32]$.

Lü et al. [27] proposed a hybrid metaheuristic approach (HMA) which combines a basic tabu search procedure and the genetic search framework. HMA is characterized by its diversification-guided recombination operator and qualityand-distance-based population updating strategy. The dedicated recombination operator aims to generate diversified offspring solutions in order to explore new promising search regions while the tabu search procedure is responsible for intensified examination around the offspring solutions. Computational results showed HMA is among the current best performing procedures on the UBQP benchmark instances.

\section{Path Relinking Algorithm}

\subsection{Main Framework}

Algorithm 1 shows the path relinking procedure for UBQP. It starts with the creation of an initial set of $b$ elite solutions RefSet (line 4, see Sect. 3.2) and identifies the best and worst solutions in RefSet in terms of the objective function value for the purpose of updating RefSet (line 5). For each elite solution $x_{i} \in$ RefSet, a binary value $\operatorname{Tag}(i)$ indicates whether $x_{i}$ can take part in a relinking process. Initially, assigning each solution in Ref Set a TRUE Tag which becomes FALSE when it is selected as the initiating solution or the guiding solution. The set PairSet contains the index pairs $(i, j)$ designating the initiating and guiding solution from Ref Set used for the relinking process. PairSet is initially composed of all the index pairs $(i, j)$ such that at least one corresponding Tag has the value TRUE (line 7). As soon as PairSet is constructed, all the Tag are marked FALSE (line 8). 


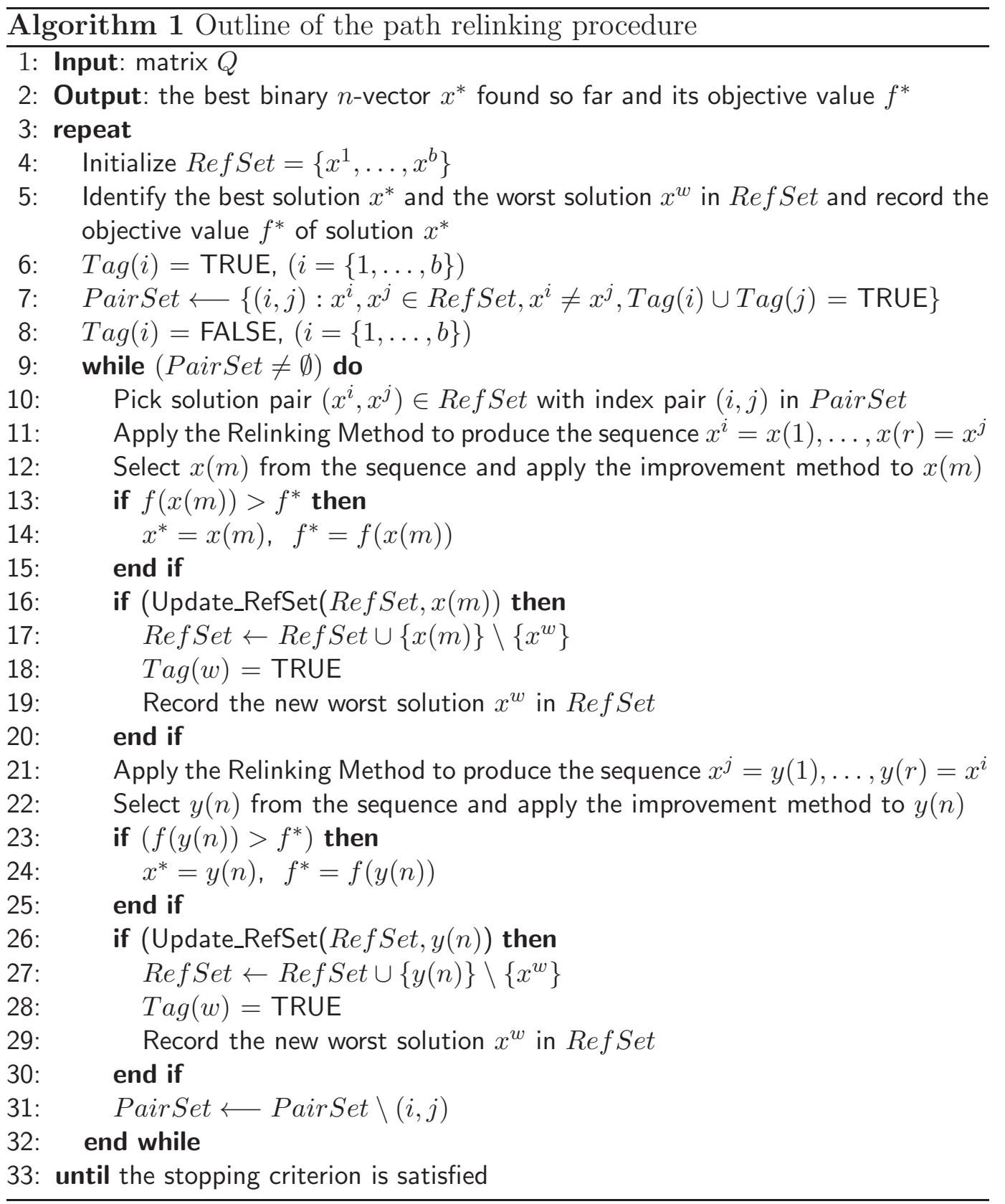

The inner while loop (lines 9-32) generates new solutions by building paths for each pair of solutions of PairSet and updates RefSet with specific new solutions. First, one index pair $(i, j)$ is selected from PairSet according to lexicographical order (line 10) to designate two solutions $x^{i}, x^{j} \in \operatorname{RefSet}$. The Relinking Method is then applied to these two solutions to generate two paths connecting $x^{i}$ and $x^{j}$ (lines 11, 21, see Sect. 3.5). Secondly, one solution $x(m)$ on each path is selected to be further improved by the Improvement Method (lines 12, 22, see Sect. 3.3). The next step tests Update_RefSet to decide if the new improved solution is used to update Ref Set (lines 16, 26, see Sect. 3.4). If the update is confirmed, the new solution is inserted in RefSet to replace the worst solution $x^{w}$ with its Tag set to be TRUE (lines 16-18, 
26-28, see Sect. 3.4). The current selected pair $(i, j)$ is then deleted from the set PairSet (line 31). This while-loop procedure continues until all the pairs in Pair Set are examined, i.e., Pair Set becomes empty.

Our path relinking algorithm has the following characteristics. First, considering the path generation procedure, each solution pair originating from Ref Set undergoes a relinking phase and two paths are considered for each pair $\left(x^{i}, x^{j}\right)$ : one from $x^{i}$ to $x^{j}$ and the other from $x^{j}$ to $x^{i}$. Secondly, each new high-quality solution derived by path relinking is a candidate to take part in a subsequent relinking process as an initiating or guiding solution, using a probabilistic selection process that assures the solution will eventually get selected. Thirdly, upon the completion of the path relinking phase that ultimately examines all pairs of solutions in RefSet, we rebuild RefSet to restart the path-relinking procedure, and repeat this restarting process until the stopping criterion is satisfied.

\subsection{The RefSet Initialization Method}

The initial RefSet contains $b$ different locally optimal solutions and is constructed as follows. Starting from scratch, we randomly assign a value of 0 or 1 to each variable to produce an initial solution, and then subject this solution to our improvement method to obtain a local optimum (see Sect. 3.3). The resulting improved solution is added to RefSet if it does not duplicate any solution currently in RefSet. This procedure is repeated until the size of Ref Set reaches the cardinality $b$.

When PairSet becomes empty, RefSet is recreated. The best solution $x^{*}$ previously found becomes a member of the new Ref Set and the remaining solutions are generated in the same way as in constructing RefSet in the first round.

\subsection{The Improvement Method}

The improvement method employs a basic tabu search procedure that is implemented in the same way as the tabu search component of the hybrid metaheuristic approach (HMA) [27]. Specifically, it employs a simple one-flip move neighborhood, which consists of changing (flipping) the value of a single variable $x_{i}$ to its complementary value $1-x_{i}$. Each time a move is carried out, the reverse move is forbidden for the next TabuTenure iterations [13]. In practice, we elected to set the tabu tenure by TabuTenure $(i)=t t c+\operatorname{rand}(10)$, where $t t c$ is a selected constant and rand(10) takes a random value from 1 to 10 . Once a move is performed, we update a subset of move values affected by the move using a fast incremental evaluation technique introduced in [18]. 
Accompanying this rule, a simple aspiration criterion is applied that permits a move to be selected in spite of being tabu if it leads to a solution better than the current best solution. By convention we speak of "better" and "best" in relation to the objective function value $f(x)$. (Similarity, we refer to the objective function value when speaking of solution quality.) The TS procedure stops when the best solution cannot be improved within a given number $\mu$ of moves that called improvement cutoff.

\subsection{The RefSet Update Method}

The updating procedure of Ref Set is invoked each time a newly constructed solution is improved by tabu search. The improved solution is permitted to be added into RefSet if it is distinct from any solution in Ref Set and better than the worst solution $x^{w}$ in RefSet. Once this condition is satisfied, the worst solution $x^{w}$ is replaced by the improved solution and the position $w$ is indicated as referring to a new solution.

\subsection{The Relinking Method}

The relinking method is used to generate new solutions by exploring trajectories (strictly confined to the neighborhood space) that connect high-quality solutions. The solution that begins the path is called the initiating solution while the solution that the path leads to is called the guiding solution [1517]. We propose two ways to generate such a path: One is based on a dedicated greedy function (whose evaluations are given by the objective function of UBQP problem) while the other operates in a random manner. Algorithms 2 and 3 describe these two methods in details.

In order to describe our relinking procedure, we first give some primary definitions, denoting the initiating solution by $x^{i}$ and the guiding solution by $x^{j}$ :

- $N C$ : the set of variable indices for which $x^{i}$ and $x^{j}$ have different values.

- $\Delta_{t}$ : a vector that stores the objective value deviation of the current solution from the resulting solution after flipping the $t$ th variable.

- $P V$ : the path vector that stores the selected flip variable at each step throughout the transiting from $x^{i}$ to $x^{j}$ (Consequently, by knowing either the initiating solution or the current terminal solution, each solution generated on the path can be recovered by referring to $P V)$.

- $F I$ : a vector that records the difference $f(x)-f\left(x^{i}\right)$ for each solution $x$ generated when transiting from $x^{i}$ to $x^{j}$. 


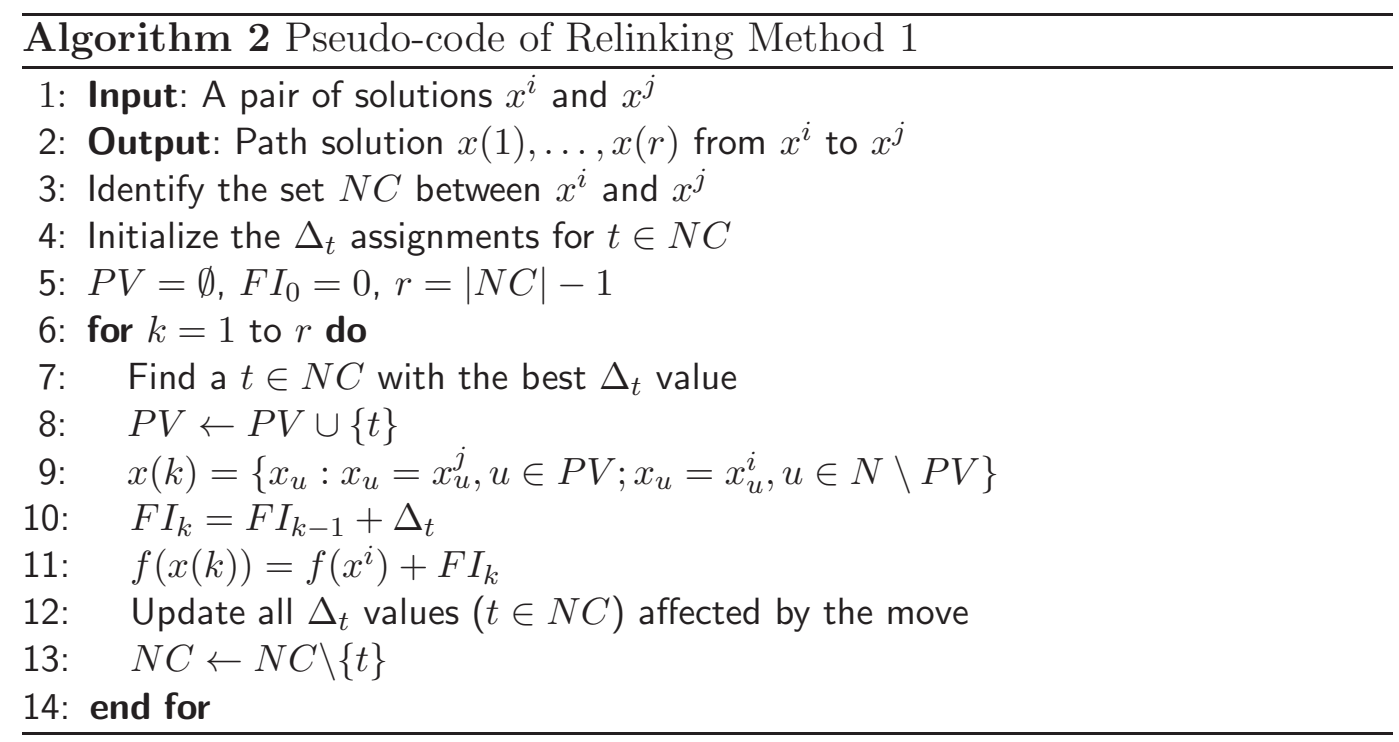

Algorithm 2 shows the first relinking method. Initially, we identify the set $N C$ of variables whose values differ between the initiating solution and the guiding solution. The $\Delta$ value of each element in $N C$ is also precalculated. At each step toward the guiding solution, we select the variable with the best $\Delta$ value and then add it into the path vector $P V$. Moreover, we record the current increment $F I$ value and the objective value $f(x)$ of the current generated solution $x$. Finally, the vector $\Delta$ is updated using the fast incremental evaluation technique of [18]. Since two adjacent solutions on the path differ from each other in the assignment of only one variable, this relinking procedure accomplishes the path construction from the initiating solution to the guiding solution after exactly $|N C|-1$ steps.

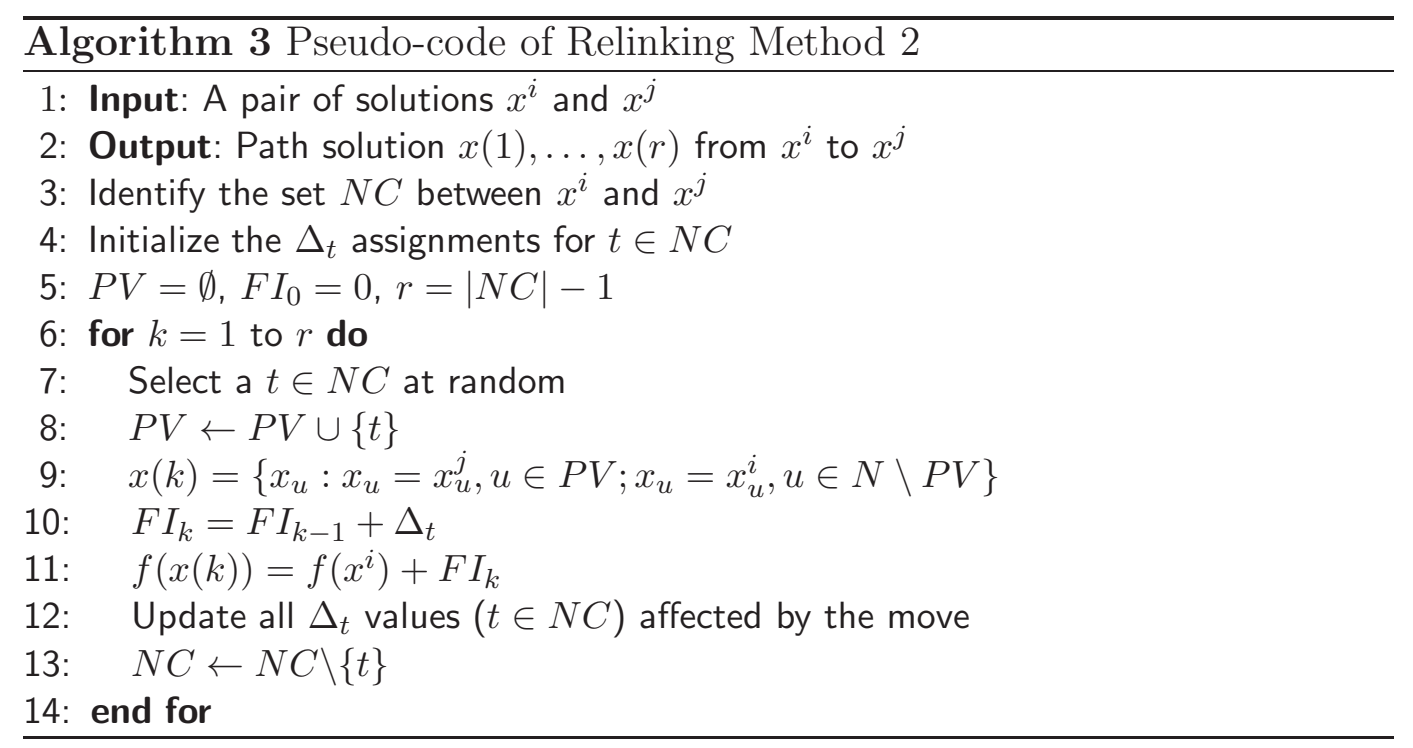

The second relinking method, shown in Algorithm 3, is based on the rule of selecting an element in $N C$ randomly at each step (line 7). The remained components of the method are the same as in Algorithm 2. 


\subsection{Path Solution Selection}

Since two consecutive solutions on a relinking path differ only by flipping a single variable, it is not productive to apply an improvement method to each solution on the path since many of these solutions would lead to the same local optimum. In addition, the improvement method is a time-consuming process, so we restrict its use to being applied to only a single solution on the path, which we select by reference both to its solution quality and to the hamming distance of this solution to the initiating and guiding solutions. Specifically, we set up a candidate solution list (CSL), consisting of the path solutions having a distance of at least $\gamma \cdot|N C|$ from both the initiating and guiding solutions (where $\gamma \in(0,1]$ is a parameter). The solution with the highest quality in CSL is picked for further amelioration by the improvement method.

\section{Computational Results}

In this section, we report extensive computational results of our two path relinking algorithms on a large collection of various benchmark instances and compare our results with those of several state-of-the-art methods in the literatures.

\subsection{Test Instances}

Five sets of test problems are considered in the experiments, amounting to 134 instances. The first set of benchmarks is composed of 10 largest instances of size $n=2500$ from the ORLIB [3]. They all have a density of 0.1 and are named by b2500.1,..,b2500.10. These instances are frequently used in the literature by many authors, see for instance $[4,22,30-32,34,19,27]$.

The second set of benchmarks consists of 21 randomly generated large problem instances named p3000.1,..,p7000.3 with sizes ranging from $n=3000$ to 7000 and with densities from 0.5 to $1.0 .{ }^{1}$ Experiments reported in $[32,34,19,27,37,38]$ show that these large instances are particularly challenging UBQP problems, especially in the case of instances with more than 5000 variables.

The third set of benchmarks includes 69 instances derived from the MaxCut problem, named G1,..,G72, with variable sizes ranging from $n=800$ to

\footnotetext{
1 The sources of the generator and input files to replicate these problem instances
} can be found at: http://www.soften.ktu.lt/ gintaras/ubqop_its.html 
Table 1

Settings of Important Parameters

\begin{tabular}{lllll}
\hline \multirow{2}{*}{ Parameters } & \multirow{2}{*}{ Section } & \multirow{2}{*}{ Description } & \multicolumn{2}{c}{ Values } \\
\cline { 4 - 5 } & & & UBQP & MaxCut \\
\hline$b$ & 3.2 & RefSet size & 10 & 10 \\
$t t c$ & 3.3 & tabu tenure constant & $\mathrm{n} / 100$ & $\mathrm{n} / 10$ \\
$\mu$ & 3.3 & improvement cutoff of TS & $5 \mathrm{n}$ & 10000 \\
$\gamma$ & 3.6 & distance scale & $1 / 3$ & $1 / 3$ \\
\hline
\end{tabular}

10000. ${ }^{2}$ These instances are created by using a machine-independent graph generator, composed of toroidal, planar and random weighted graphs with weight values 1,0 or -1 . The first 54 instances have been employed by numerous authors to test their algorithms $[8,12,29,33,36]$ and the results for the remaining 15 larger instances are reported in [10].

The fourth set of benchmarks contains 30 instances with size $n=128$ (named G54100,..,G541000), $n=1000$ (named G10100,..,G101000) and $n=2744$ (named G14100,...,G141000), respectively. ${ }^{3}$ These instances are created from cubic lattices modeling Ising spin glasses with weight values 1, 0 or -1. Computational results on these instances were reported in $[8,12,29,33,36]$.

The last set is composed of 4 DIMACS instances containing from 512 to 3375 vertices and 1536 to 10125 edges. ${ }^{4}$

\subsection{Experimental Protocol}

Our path relinking $(\mathrm{PR})$ algorithms are programmed in $\mathrm{C}$ and compiled using GNU GCC on a PC running Windows XP with Pentium $2.83 \mathrm{GHz} \mathrm{CPU}$ and 8GB RAM. The computational results reported in this section were obtained with the parameter values shown in Table 1, where the last two columns respectively denote the settings for the 31 random UBQP instances and the 103 MaxCut instances. Given the stochastic nature of our PR algorithms, each instance is independently solved 20 times by each algorithm.

\subsection{Computational Results on the Random UBQP Instances}

Our first experiment undertakes to evaluate the PR algorithms on the 31 random instances with 2500 to 7000 variables (the first two sets of benchmarks). The results are summarized in Tables 2 and 3. Our algorithms use CPU clock time to give the stopping condition subject to having completed at least one

\footnotetext{
2 http://www.stanford.edu/ yyye/yyye/Gset

3 http://www.optsicom.es/maxcut/\#instances

4 http://dimacs.rutgers.edu/Challenges/Seventh/Instances/
} 
Table 2

Computational Results on Beasley Instances

\begin{tabular}{|c|c|c|c|c|c|c|c|}
\hline \multirow{2}{*}{ Instance } & \multirow{2}{*}{$f_{\text {prev }}$} & \multicolumn{3}{|c|}{ PR1 } & \multicolumn{3}{|c|}{ PR2 } \\
\hline & & $f_{\text {best }}$ & $g_{a v r}$ & time & $f_{\text {best }}$ & $g_{a v r}$ & time \\
\hline b2500.1 & 1515944 & 1515944 & 0.0 & 11 & 1515944 & 0.0 & 14 \\
\hline b2500.2 & 1471392 & 1471392 & 0.0 & 101 & 1471392 & 58.4 & 102 \\
\hline b2500.3 & 1414192 & 1414192 & 13.4 & 49 & 1414192 & 0.0 & 36 \\
\hline b2500.4 & 1507701 & 1507701 & 0.0 & 6 & 1507701 & 0.0 & 7 \\
\hline b2500.5 & 1491816 & 1491816 & 0.0 & 14 & 1491816 & 0.0 & 18 \\
\hline b2500.6 & 1469162 & 1469162 & 0.0 & 25 & 1469162 & 0.0 & 23 \\
\hline b2500.7 & 1479040 & 1479040 & 0.0 & 48 & 1479040 & 0.0 & 50 \\
\hline b2500.8 & 1484199 & 1484199 & 0.0 & 20 & 1484199 & 0.0 & 16 \\
\hline b2500.9 & 1482413 & 1482413 & 0.0 & 51 & 1482413 & 0.0 & 103 \\
\hline b2500.10 & 1483355 & 1483355 & 0.0 & 55 & 1483355 & 0.0 & 75 \\
\hline Average & & & 1.34 & 38 & & 5.84 & 44.4 \\
\hline
\end{tabular}

round of the PR procedure. The time limit for 10 ORLIB instances for a single run is set to be 1 minute and for the 21 larger random instances with 3000 , 4000, 5000, 6000 and 7000 variables is set at 5, 10, 20, 30 and 50 minutes. This time cutoff is the same as in $[27,32,34]$.

Tables 2 and 3 respectively show the computational statistics of applying our PR1 and PR2 algorithms to the 10 ORLIB instances and the 21 large random instances. In both tables, columns 1 and 2 respectively give the instance names and the previous best objective values $f_{\text {prev }}$. These best values were first reported in $[32,34]$ and recently improved in [19]. The columns under heading PR1 and PR2 list: the best objective value $f_{\text {best }}$, the average objective gap to the previous best objective values $g_{\text {avr }}$ (i.e., $f_{\text {prev }}-f_{\text {avr }}$ ) (where $f_{\text {avr }}$ represents the average objective value over 20 runs) and the average CPU time in seconds denoted by time for reaching the best objective values $f_{\text {best }}$ over 20 runs. Furthermore, the last row "Average" indicates the summary of our algorithm's average performance.

Table 2 discloses that both PR1 and PR2 can stably reach all the previous best objective values for the 10 largest Beasley instances. Moreover, PR1 performs slightly better than PR2 when it comes to the criteria of $g_{\text {avr }}$ and time to the previous best result $f_{\text {prev }}$. Table 3 indicates that on the 21 large and difficult random instances, PR1 produced the same results as PR2 given that both can reach the previous best known objective values for all of the tested instances. However, PR1 is superior to PR2 in terms of the average gap (457.1 versus 690.4) although the CPU time to obtain the best solution is slightly longer, (749.2 versus 665.3 seconds).

In order to further evaluate our PR1 and PR2 algorithms, we compare our results with those obtained from some of best performing algorithms in the literature. For this purpose, we restrict our attention to comparisons with 5 methods that have reported the best results for the most challenging problems. These methods are respectively named ITS [34], MST2 [32], SA [22], D²TS [19] and HMA [27]. The results for the first 3 of these reference algorithms are 
Table 3

Computational Results on Palubeckis Instances

\begin{tabular}{|c|c|c|c|c|c|c|c|}
\hline \multirow{2}{*}{ Instance } & \multirow{2}{*}{$f_{\text {prev }}$} & \multicolumn{3}{|c|}{ PR1 } & \multicolumn{3}{|c|}{ PR2 } \\
\hline & & $f_{\text {best }}$ & $g_{a v r}$ & time & $f_{\text {best }}$ & $g_{a v r}$ & time \\
\hline p3000.1 & 3931583 & 3931583 & 0.0 & 85 & 3931583 & 80.4 & 81 \\
\hline p3000.2 & 5193073 & 5193073 & 0.0 & 68 & 5193073 & 0.0 & 64 \\
\hline p3000.3 & 5111533 & 5111533 & 35.8 & 115 & 5111533 & 71.7 & 155 \\
\hline p3000.4 & 5761822 & 5761822 & 0.0 & 56 & 5761822 & 0.0 & 97 \\
\hline p3000.5 & 5675625 & 5675625 & 90.2 & 162 & 5675625 & 278.5 & 226 \\
\hline $\mathrm{p} 4000.1$ & 6181830 & 6181830 & 0.0 & 125 & 6181830 & 0.0 & 159 \\
\hline p4000.2 & 7801355 & 7801355 & 71.2 & 456 & 7801355 & 313.5 & 302 \\
\hline p4000.3 & 7741685 & 7741685 & 0.0 & 295 & 7741685 & 63.9 & 436 \\
\hline p4000.4 & 8711822 & 8711822 & 0.0 & 277 & 8711822 & 0.0 & 392 \\
\hline $\mathrm{p} 4000.5$ & 8908979 & 8908979 & 490.8 & 272 & 8908979 & 385.1 & 327 \\
\hline p5000.1 & 8559680 & 8559680 & 611.8 & 623 & 8559680 & 918.0 & 387 \\
\hline p5000.2 & 10836019 & 10836019 & 620.3 & 821 & 10836019 & 498.7 & 609 \\
\hline p5000.3 & 10489137 & 10489137 & 995.4 & 1285 & 10489137 & 317.5 & 967 \\
\hline p5000.4 & 12252318 & 12252318 & 1257.7 & 760 & 12252318 & 1168.4 & 767 \\
\hline p5000.5 & 12731803 & 12731803 & 51.3 & 676 & 12731803 & 166.3 & 726 \\
\hline p6000.1 & 11384976 & 11384976 & 201.0 & 1820 & 11384976 & 822.4 & 1136 \\
\hline p6000.2 & 14333855 & 14333855 & 221.1 & 1391 & 14333855 & 576.8 & 1076 \\
\hline p6000.3 & 16132915 & 16132915 & 1743.5 & 1128 & 16132915 & 2017.3 & 1053 \\
\hline p7000.1 & 14478676 & 14478676 & 935.4 & 2275 & 14478676 & 1523.1 & 1917 \\
\hline p7000.2 & 18249948 & 18249948 & 1942.4 & 1793 & 18249948 & 2986.1 & 1591 \\
\hline p7000.3 & 20446407 & 20446407 & 331.9 & 1251 & 20446407 & 2310.5 & 1503 \\
\hline Average & & & 457.1 & 749.2 & & 690.4 & 665.3 \\
\hline
\end{tabular}

Table 4

Best Results Comparison on Palubeckis Instances

\begin{tabular}{ccccccccc}
\hline \multirow{2}{*}{ Instance } & \multirow{2}{*}{$f_{\text {prev }}$} & \multicolumn{7}{c}{ best solution gap (i.e., $f_{\text {prev }}-f_{\text {best }}$ ) } \\
\cline { 2 - 8 } & PR1 & PR2 & ITS $[34]$ & MST2 $[32]$ & SA $[22]$ & D $^{2}$ TS $[19]$ & HMA $[27]$ \\
\hline p5000.1 & 8559680 & 0 & 0 & 700 & 325 & 1432 & 325 & 0 \\
p5000.2 & 10836019 & 0 & 0 & 0 & 582 & 582 & 0 & 0 \\
p5000.3 & 10489137 & 0 & 0 & 0 & 0 & 354 & 0 & 0 \\
p5000.4 & 12252318 & 0 & 0 & 934 & 1643 & 444 & 0 & 0 \\
p5000.5 & 12731803 & 0 & 0 & 0 & 0 & 1025 & 0 & 0 \\
p6000.1 & 11384976 & 0 & 0 & 0 & 0 & 430 & 0 & 0 \\
p6000.2 & 14333855 & 0 & 0 & 88 & 0 & 675 & 0 & 0 \\
p6000.3 & 16132915 & 0 & 0 & 2729 & 0 & 0 & 0 & 0 \\
p7000.1 & 14478676 & 0 & 0 & 340 & 1607 & 2579 & 0 & 0 \\
p7000.2 & 18249948 & 0 & 0 & 1651 & 2330 & 5552 & 104 & 0 \\
p7000.3 & 20446407 & 0 & 0 & 0 & 0 & 2264 & 0 & 0 \\
\hline Average & & 0 & 0 & 585.6 & 589.7 & 1394.3 & 39 & 0 \\
\hline
\end{tabular}

directly extracted from [34] and those for $\mathrm{D}^{2} \mathrm{TS}$ and HMA come from [19,27].

Tables 4 and 5 show the best solution gap and average solution gap to the best known objective value of the 7 algorithms used for comparison, including PR1 and PR2. In these two tables, the last row presents the averaged results over the listed instances. Note that the results of all these algorithms are obtained almost under the same time limit. Since best known values can be easily reached for the small size instances by all these state-of-the art algorithms, we only list larger instances, consisting of 11 instances in Table 4 and 21 instances in Table 5. 
Table 5

Average Results Comparison on Palubeckis Instances

\begin{tabular}{|c|c|c|c|c|c|c|c|c|}
\hline \multirow{2}{*}{ Instance } & \multirow[b]{2}{*}{$f_{\text {prev }}$} & \multicolumn{7}{|c|}{ average solution gap (i.e., $f_{\text {prev }}-f_{\text {avr }}$ ) } \\
\hline & & PR1 & PR2 & ITS [34] & MST2 [32] & SA [22] & $\mathrm{D}^{2} \mathrm{TS}[19]$ & HMA [27] \\
\hline p3000.1 & 3931583 & 0 & 80 & 0 & 0 & 0 & 0 & 0 \\
\hline p3000.2 & 5193073 & 0 & 0 & 97 & 97 & 97 & 0 & 0 \\
\hline p3000.3 & 5111533 & 36 & 72 & 344 & 287 & 535 & 0 & 33 \\
\hline p3000.4 & 5761822 & 0 & 0 & 154 & 77 & 308 & 0 & 0 \\
\hline p3000.5 & 5675625 & 90 & 279 & 501 & 382 & 459 & 0 & 145 \\
\hline p4000.1 & 6181830 & 0 & 0 & 0 & 0 & 734 & 0 & 0 \\
\hline $\mathrm{p} 4000.2$ & 7801355 & 71 & 314 & 1285 & 804 & 1887 & 0 & 142 \\
\hline p4000.3 & 7741685 & 0 & 64 & 471 & 1284 & 79 & 0 & 6 \\
\hline $\mathrm{p} 4000.4$ & 8711822 & 0 & 0 & 438 & 667 & 536 & 0 & 38 \\
\hline p4000.5 & 8908979 & 491 & 385 & 572 & 717 & 984 & 0 & 546 \\
\hline p5000.1 & 8559680 & 612 & 918 & 971 & 581 & 2455 & 656 & 507 \\
\hline p5000.2 & 10836019 & 620 & 499 & 1068 & 978 & 2101 & 12533 & 512 \\
\hline p5000.3 & 10489137 & 995 & 318 & 1266 & 1874 & 2451 & 12876 & 332 \\
\hline p5000.4 & 12252318 & 1258 & 1168 & 1952 & 2570 & 1134 & 1962 & 1228 \\
\hline p5000.5 & 12731803 & 51 & 166 & 835 & 1233 & 1172 & 239 & 284 \\
\hline p6000.1 & 11384976 & 201 & 822 & 57 & 34 & 2248 & 0 & 140 \\
\hline p6000.2 & 14333855 & 221 & 577 & 1709 & 1269 & 2067 & 1286 & 526 \\
\hline p6000.3 & 16132915 & 1744 & 2017 & 3064 & 2673 & 3845 & 787 & 2311 \\
\hline p7000.1 & 14478676 & 935 & 1523 & 1139 & 2515 & 5504 & 2138 & 819 \\
\hline p7000.2 & 18249948 & 1942 & 2986 & 4301 & 3814 & 7837 & 8712 & 1323 \\
\hline p7000.3 & 20446407 & 332 & 2311 & 3078 & 7868 & 8978 & 2551 & 1386 \\
\hline Average & & 457.1 & 690.4 & 1109.6 & 1415.4 & 2162.4 & 2082.9 & 489.4 \\
\hline
\end{tabular}

Table 4 indicates that both PR1 and PR2 outperform ITS, MST2 and SA in terms of the best solution values. PR1 and PR2 achieve the best known results for the 11 most challenging instances while ITS, MST2, SA fail for 5, 5, 10 out of 11 instances. In addition, $\mathrm{D}^{2} \mathrm{TS}$ performs slightly worse since it fails to reach the best known result for one instance p7000.2. However, it is difficult to conclude which algorithm among PR1, PR2 and HMA performs the best based on the evaluation criterion of the best solution found.

In order to further discriminate among the compared algorithms, Table 5 presents the average solution gap to the best known value of each algorithm. Firstly, we notice that over the first 10 instances with 3000 and 4000 variables, $\mathrm{D}^{2} \mathrm{TS}$ outperforms all the other 6 compared algorithms with an average gap of 0 to the best known values, meaning that $\mathrm{D}^{2} \mathrm{TS}$ is quite robust over 20 runs for these 10 instances. PR1 and PR fail to reach the gap of 0 for 4 and 6 instances, respectively. Secondly, considering the overall set of 21 instances, we find that PR1 performs the best with a gap of 457.1. HMA performs slightly worse than PR1 with a gap of 489.4. PR2 takes the third place with a gap of 690.4. In conclusion, this experiment demonstrates that both PR1 and PR2 also perform quite well with regard to the average solution quality. 
Table 6

Computational Results on small and medium MaxCut Instances of Set1

\begin{tabular}{|c|c|c|c|c|c|c|c|c|c|c|c|}
\hline \multirow{2}{*}{ Instance } & \multirow[b]{2}{*}{$f_{\text {prev }}$} & \multicolumn{3}{|c|}{ PR1 } & \multicolumn{3}{|c|}{ PR2 } & \multicolumn{2}{|c|}{ SS [29] } & \multicolumn{2}{|c|}{ CirCut [8] } \\
\hline & & $f_{\text {best }}$ & $f_{\text {avr }}$ & $\overline{\text { time }}$ & $f_{\text {best }}$ & $f_{\text {avr }}$ & $\overline{\text { time }}$ & $f_{\text {best }}$ & time & $f_{\text {best }}$ & time \\
\hline G1 & 11624 & 11624 & 11624.0 & 2 & 11624 & 11624.0 & 1 & 11624 & 139 & 11624 & 352 \\
\hline G2 & 11620 & 11620 & 11620.0 & 6 & 11620 & 11620.0 & 9 & 11620 & 167 & 11617 & 283 \\
\hline G3 & 11622 & 11620 & 11620.0 & 17 & 11620 & 11620.0 & 2 & 11622 & 180 & 11622 & 330 \\
\hline G4 & 11646 & 11646 & 11646.0 & 3 & 11646 & 11646.0 & 2 & 11646 & 194 & 11641 & 524 \\
\hline G5 & 11631 & 11631 & 11631.0 & 3 & 11631 & 11631.0 & 4 & 11631 & 205 & 11627 & 1128 \\
\hline G6 & 2178 & 2178 & 2178.0 & 9 & 2178 & 2178.0 & 6 & 2165 & 176 & 2178 & 947 \\
\hline G7 & 2003 & 2006 & 2006.0 & 2 & 2006 & 2006.0 & 7 & 1982 & 176 & 2003 & 867 \\
\hline G8 & 2003 & 2005 & 2005.0 & 8 & 2005 & 2005.0 & 6 & 1986 & 195 & 2003 & 931 \\
\hline G9 & 2048 & 2054 & 2054.0 & 16 & 2054 & 2054.0 & 10 & 2040 & 158 & 2048 & 943 \\
\hline G10 & 1994 & 2000 & 2000.0 & 22 & 2000 & 1999.8 & 29 & 1993 & 210 & 1994 & 881 \\
\hline G11 & 564 & 564 & 564.0 & 4 & 564 & 564.0 & 1 & 562 & 172 & 560 & 74 \\
\hline G12 & 556 & 556 & 556.0 & 17 & 556 & 556.0 & 15 & 552 & 242 & 552 & 58 \\
\hline G13 & 582 & 582 & 582.0 & 28 & 582 & 582.0 & 22 & 578 & 228 & 574 & 62 \\
\hline G14 & 3064 & 3063 & 3062.1 & 44 & 3064 & 3062.6 & 1188 & 3060 & 187 & 3058 & 128 \\
\hline G15 & 3050 & 3050 & 3049.3 & 49 & 3050 & 3049.3 & 51 & 3049 & 143 & 3049 & 155 \\
\hline G16 & 3052 & 3052 & 3051.3 & 27 & 3052 & 3051.4 & 47 & 3045 & 162 & 3045 & 142 \\
\hline G17 & 3043 & 3047 & 3045.5 & 235 & 3047 & 3046.4 & 110 & 3043 & 313 & 3037 & 366 \\
\hline G18 & 988 & 992 & 992.0 & 16 & 992 & 992.0 & 12 & 988 & 174 & 978 & 497 \\
\hline G19 & 903 & 906 & 906.0 & 11 & 906 & 906.0 & 14 & 903 & 128 & 888 & 507 \\
\hline G20 & 941 & 941 & 941.0 & 13 & 941 & 941.0 & 9 & 941 & 191 & 941 & 503 \\
\hline G21 & 931 & 931 & 931.0 & 11 & 931 & 931.0 & 19 & 930 & 233 & 931 & 524 \\
\hline G22 & 13359 & 13359 & 13353.5 & 1652 & 13359 & 13354.5 & 943 & 13346 & 1336 & 13346 & 493 \\
\hline G23 & 13342 & 13342 & 13333.0 & 517 & 13342 & 13331.6 & 879 & 13317 & 1022 & 13317 & 457 \\
\hline G24 & 13337 & 13337 & 13327.3 & 1257 & 13333 & 13325.3 & 1876 & 13303 & 1191 & 13314 & 521 \\
\hline G25 & 13326 & 13338 & 13328.0 & 957 & 13339 & 13328.2 & 1078 & 13320 & 1299 & 13326 & 1600 \\
\hline G26 & 13314 & 13324 & 13313.7 & 710 & 13326 & 13312.3 & 333 & 13294 & 1415 & 13314 & 1569 \\
\hline G27 & 3318 & 3337 & 3327.3 & 851 & 3336 & 3326.9 & 753 & 3318 & 1438 & 3306 & 1456 \\
\hline G28 & 3285 & 3296 & 3286.0 & 1723 & 3296 & 3288.9 & 1512 & 3285 & 1314 & 3260 & 1543 \\
\hline G29 & 3389 & 3404 & 3395.2 & 861 & 3405 & 3391.9 & 1618 & 3389 & 1266 & 3376 & 1512 \\
\hline G30 & 3403 & 3412 & 3404.6 & 1655 & 3411 & 3404.8 & 843 & 3403 & 1196 & 3385 & 1463 \\
\hline G31 & 3288 & 3306 & 3299.7 & 624 & 3306 & 3299.5 & 752 & 3288 & 1336 & 3285 & 1448 \\
\hline G32 & 1410 & 1408 & 1400.9 & 893 & 1410 & 1404.6 & 450 & 1398 & 901 & 1390 & 221 \\
\hline G33 & 1382 & 1382 & 1373.9 & 1019 & 1382 & 1376.1 & 986 & 1362 & 926 & 1360 & 198 \\
\hline G34 & 1384 & 1382 & 1375.4 & 1608 & 1384 & 1378.2 & 1747 & 1364 & 950 & 1368 & 237 \\
\hline G35 & 7684 & 7674 & 7663.3 & 1372 & 7679 & 7670.8 & 959 & 7668 & 1258 & 7670 & 440 \\
\hline G36 & 7677 & 7666 & 7653.1 & 316 & 7671 & 7658.7 & 1790 & 7660 & 1392 & 7660 & 400 \\
\hline G37 & 7689 & 7673 & 7663.3 & 1736 & 7682 & 7667.9 & 965 & 7664 & 1387 & 7666 & 382 \\
\hline G38 & 7681 & 7674 & 7663.4 & 614 & 7682 & 7670.4 & 1775 & 7681 & 1012 & 7646 & 1189 \\
\hline G39 & 2395 & 2402 & 2391.3 & 526 & 2407 & 2391.1 & 1588 & 2393 & 1311 & 2395 & 852 \\
\hline G40 & 2387 & 2394 & 2381.2 & 1748 & 2399 & 2383.3 & 879 & 2374 & 1166 & 2387 & 901 \\
\hline G41 & 2398 & 2402 & 2380.0 & 1181 & 2404 & 2388.9 & 529 & 2386 & 1017 & 2398 & 942 \\
\hline G42 & 2469 & 2475 & 2462.3 & 1177 & 2478 & 2466.2 & 1575 & 2457 & 1458 & 2469 & 875 \\
\hline G43 & 6660 & 6660 & 6660.0 & 22 & 6660 & 6659.9 & 19 & 6656 & 406 & 6656 & 213 \\
\hline G44 & 6650 & 6650 & 6649.9 & 18 & 6650 & 6649.9 & 32 & 6648 & 356 & 6643 & 192 \\
\hline G45 & 6654 & 6654 & 6653.9 & 43 & 6654 & 6653.9 & 50 & 6642 & 354 & 6652 & 210 \\
\hline G46 & 6645 & 6649 & 6648.2 & 18 & 6649 & 6648.8 & 36 & 6634 & 498 & 6645 & 639 \\
\hline G47 & 6656 & 6657 & 6656.6 & 99 & 6657 & 6656.8 & 20 & 6649 & 359 & 6656 & 633 \\
\hline G48 & 6000 & 6000 & 6000.0 & 3 & 6000 & 6000.0 & 3 & 6000 & 20 & 6000 & 119 \\
\hline G49 & 6000 & 6000 & 6000.0 & 3 & 6000 & 6000.0 & 2 & 6000 & 35 & 6000 & 134 \\
\hline G50 & 5880 & 5880 & 5880.0 & 2 & 5880 & 5880.0 & 2 & 5880 & 27 & 5880 & 231 \\
\hline G51 & 3846 & 3848 & 3844.6 & 312 & 3848 & 3846.4 & 158 & 3846 & 513 & 3837 & 497 \\
\hline G52 & 3849 & 3851 & 3847.6 & 610 & 3851 & 3848.4 & 373 & 3849 & 551 & 3833 & 507 \\
\hline G53 & 3846 & 3849 & 3846.9 & 151 & 3850 & 3847.7 & 88 & 3846 & 424 & 3842 & 503 \\
\hline G54 & 3846 & 3852 & 3848.6 & 522 & 3851 & 3847.8 & 318 & 3846 & 429 & 3842 & 524 \\
\hline Average & & & & 469.3 & & & 490.6 & & 621.0 & & 616.7 \\
\hline Better & & 24 & & & 25 & & & 0 & & 0 & \\
\hline Equal & & 22 & & & 24 & & & 22 & & 20 & \\
\hline Worse & & 8 & & & 5 & & & 32 & & 34 & \\
\hline
\end{tabular}




\subsection{Computational Results on the MaxCut Instances}

In this section, we test our PR algorithms on 3 sets of benchmarks with a total of 103 instances derived from MaxCut problem. In Tables 6-9, columns 1 and 2 respectively give the instance name and the previous best solution value $f_{\text {prev }}$ from references $[8,29,33,36]$ which are dedicated MaxCut algorithms. The columns under the headings PR1 and PR2 list the best objective value $f_{\text {best }}$, the average objective value $f_{\text {avr }}$ and the CPU time in seconds denoted by time for reaching the best results $f_{\text {best }}$. The columns under the headings SS and CirCut report the best objective value $f_{\text {best }}$ and the required CPU time to reach $f_{\text {best }}$. We focus on comparing our algorithms with the SS and CirCut algorithms, which yield best results in the literature on many test instances. The results of SS and CirCut algorithms are directly extracted from [29]. The last three rows summarize the comparison between these algorithms and ours. The rows better, equal and worse respectively denote the number of instances for which each algorithm gets results that are better, equal and worse than the previous best known results. We mark in bold those results that are the updated best known values obtained by PR1 and PR2.

Table 6 reports the results on 54 instances of the third set of benchmarks within a time limit of 30 minutes. From this table, we first notice that our algorithms are able to find better objective values than the best known values in the literature. Meanwhile, PR2 slightly outperforms PR1 in terms of the best objective values. Specifically, PR1 can improve the previous best known objective values for 24 instances and match the previous best for 22 instances, while PR2 can improve the previous best known objective values for 25 instances and match the previous best for 24 instances. Moreover, PR1 and PR2 fail to reach the best known results for 8 and 5 instances respectively, while SS and CirCut fail on 32 and 34 instances, respectively. Additionally, PR1 and PR2 reaches its best results in a shorter CPU time than the time taken by SS and CirCut to reach their best results. These outcomes provide evidence of the efficacy of our path relinking approach.

Table 7 reports the results of 15 largest instances from the same set of benchmark as above with variables ranging from 5000 to 10000. For instances with 5000, 7000, 8000, 9000 and 10000 variables, we report the results for a time limit of 1, 2, 4, 4 and 4 hours, respectively. The previous best objective values $f_{\text {prev }}$ are cited from [10], which is the only paper, to the best of our knowledge, that reports the results on these instances. As can be seen from Table 7, both PR1 and PR2 obtain new best known results on 13 out of these 15 large instances and obtains results inferior to the best known results only on 2 instances. Moreover, PR2 outperforms PR1 by obtaining better solutions for 14 instances. 
Table 7

Computational Results on large MaxCut Instances of Set1

\begin{tabular}{ccccccccc}
\hline \multirow{2}{*}{ Instance } & $f_{\text {prev }}$ & \multicolumn{3}{c}{ PR1 } & & \multicolumn{3}{c}{ PR2 } \\
\cline { 3 - 5 } \cline { 7 - 8 } G55 & 9960 & 10253 & 10233.7 & 3996 & & $\mathbf{1 0 2 6 5}$ & 10234.0 & 3231 \\
G56 & 3649 & 3975 & 3958.0 & 3991 & & $\mathbf{3 9 8 1}$ & 3959.2 & 3842 \\
G57 & 3220 & 3448 & 3436.0 & 3656 & & $\mathbf{3 4 7 2}$ & 3462.0 & 4403 \\
G58 & - & 19183 & 19159.3 & 3979 & & $\mathbf{1 9 2 0 5}$ & 19182.0 & 3715 \\
G59 & - & $\mathbf{6 0 2 7}$ & 5989.2 & 3876 & & $\mathbf{6 0 2 7}$ & 6006.2 & 5194 \\
G60 & 13658 & 14109 & 14077.5 & 7738 & & $\mathbf{1 4 1 1 2}$ & 14091.8 & 6300 \\
G61 & 5273 & 5716 & 5688.8 & 7782 & & $\mathbf{5 7 3 0}$ & 5695.7 & 5381 \\
G62 & 4612 & 4804 & 4785.7 & 8110 & & $\mathbf{4 8 3 6}$ & 4830.2 & 6114 \\
G63 & 8059 & 26876 & 26845.8 & 4826 & & $\mathbf{2 6 9 1 6}$ & 26879.3 & 5867 \\
G64 & 7861 & 8623 & 8569.5 & 8790 & & $\mathbf{8 6 4 1}$ & 8594.1 & 6974 \\
G65 & 13286 & 5482 & 5468.7 & 16248 & & 5526 & 5515.9 & 15004 \\
G66 & - & 6272 & 6257.8 & 16031 & & $\mathbf{6 3 1 4}$ & 6302.4 & 15191 \\
G67 & - & 6856 & 6832.0 & 17213 & & $\mathbf{6 9 0 2}$ & 6884.6 & 12372 \\
G70 & 9499 & 9405 & 9378.6 & 15202 & & 9463 & 9434.0 & 14531 \\
G72 & 6644 & 6892 & 6876.2 & 14422 & $\mathbf{6 9 4 6}$ & 6933.8 & 15898 \\
\hline Better & & 13 & & & & 13 & & \\
Equal & & 0 & & & 0 & & \\
Worse & & 2 & & & & & & \\
\hline
\end{tabular}

The results of the 30 instances from the fourth set of benchmarks are shown in Table 8. For the instances with variables numbering 128, 1000 and 2744, the results are reported with a time limit of 1 second, 10 minutes and 30 minutes. Table 8 shows that our PR1 and PR2 algorithms once again outperform the two reference algorithms. Both PR1 and PR2 can match the best known results on 21 and 20 out of 30 instances, respectively. By contrast, SS and CirCut can match the previous best results on 10 instances. PR1 and PR2 fail to match the best known results on 9 and 10 out of 30 instances, respectively. By contrast, both SS and CirCut fail to match the previous best results on 20 instances.

Comparing PR1 and PR2 to each other, the PR2 algorithm achieves better results for 4 instances (G14100, G14400, G14800 and G141000) while PR1 obtain better results for 2 instances (G14300 and G14500). In addition, PR2 obtains its best solutions faster than PR1, 377.5 vs 473.2 seconds on average. We note that CirCut consumes less CPU time than ours, though the quality of its solutions does not measure up.

The results of the fifth set of benchmarks using a time limit of 30 minutes are shown in Table 9. For the instance pm3-15-50, both PR1 and PR2 are able to improve the previous best known result from a value of 3000 to the value of 3010 and 3014, respectively. For the instance pm3-8-50, PR1 and PR2 match the previously best known result but the other refered algorithms fail to do so. (We note that an algorithm fail to obtain a number of best known results and still qualify as a top performing algorithm in the literature, given that other algorithms may generally obtain still fewer best known results.) Moreover, both of our algorithms and CirCut can reach the best known result on instance 
Table 8

Computational Results on MaxCut Instances of Set2

\begin{tabular}{|c|c|c|c|c|c|c|c|c|c|c|c|}
\hline \multirow{2}{*}{ Instance } & \multirow[b]{2}{*}{$f_{\text {prev }}$} & \multicolumn{3}{|c|}{ PR1 } & \multicolumn{3}{|c|}{ PR2 } & \multicolumn{2}{|c|}{ SS [29] } & \multicolumn{2}{|c|}{ CirCut [8] } \\
\hline & & $f_{\text {best }}$ & $f_{a v r}$ & $\overline{\text { time }}$ & $f_{\text {best }}$ & $f_{\text {avr }}$ & $\overline{\text { time }}$ & $f_{\text {best }}$ & $\overline{\text { time }}$ & $f_{\text {best }}$ & $\overline{\text { time }}$ \\
\hline G54100 & 110 & 110 & 110.0 & 0 & 110 & 110.0 & 0 & 110 & 1.9 & 110 & 16.2 \\
\hline G54200 & 112 & 112 & 112.0 & 0 & 112 & 112.0 & 0 & 112 & 1.9 & 112 & 18.6 \\
\hline G54300 & 106 & 106 & 106.0 & 0 & 106 & 106.0 & 0 & 106 & 2.1 & 106 & 15.8 \\
\hline G54400 & 114 & 114 & 114.0 & 0 & 114 & 114.0 & 0 & 114 & 2.1 & 114 & 16.0 \\
\hline G54500 & 112 & 112 & 112.0 & 0 & 112 & 112.0 & 0 & 112 & 2.3 & 112 & 15.8 \\
\hline G54600 & 110 & 110 & 110.0 & 0 & 110 & 110.0 & 0 & 110 & 2.1 & 110 & 15.4 \\
\hline G54700 & 112 & 112 & 112.0 & 0 & 112 & 112.0 & 0 & 112 & 2.0 & 112 & 14.8 \\
\hline G54800 & 108 & 108 & 108.0 & 0 & 108 & 108.0 & 0 & 108 & 2.1 & 108 & 15.4 \\
\hline G54900 & 110 & 110 & 110.0 & 0 & 110 & 110.0 & 0 & 110 & 1.8 & 110 & 15.5 \\
\hline G541000 & 112 & 112 & 112.0 & 0 & 112 & 112.0 & 0 & 112 & 1.4 & 112 & 16.4 \\
\hline G10100 & 896 & 896 & 894.3 & 99 & 896 & 894.6 & 24 & 882 & 406.1 & 880 & 106.0 \\
\hline G10200 & 900 & 900 & 900.0 & 1 & 900 & 900.0 & 1 & 894 & 302.4 & 892 & 116.0 \\
\hline G10300 & 892 & 892 & 890.5 & 342 & 892 & 891.3 & 71 & 884 & 410.4 & 882 & 112.0 \\
\hline G10400 & 898 & 898 & 898.0 & 3 & 898 & 898.0 & 1 & 892 & 485.9 & 894 & 103.0 \\
\hline G10500 & 886 & 886 & 885.4 & 48 & 886 & 885.4 & 36 & 880 & 400.9 & 882 & 106.0 \\
\hline G10600 & 888 & 888 & 888.0 & 1 & 888 & 888.0 & 1 & 870 & 461.8 & 886 & 119.0 \\
\hline G10700 & 900 & 900 & 898.1 & 400 & 900 & 898.2 & 414 & 890 & 386.2 & 894 & 115.0 \\
\hline G10800 & 882 & 882 & 881.3 & 39 & 882 & 881.2 & 31 & 880 & 466.9 & 874 & 104.0 \\
\hline G10900 & 902 & 902 & 900.9 & 143 & 902 & 901.5 & 63 & 888 & 493.6 & 890 & 121.0 \\
\hline G101000 & 894 & 894 & 893.5 & 27 & 894 & 893.7 & 8 & 886 & 352.8 & 886 & 111.0 \\
\hline G14100 & 2446 & 2442 & 2437.1 & 581 & 2444 & 2437.6 & 1682 & 2428 & 1320.6 & 2410 & 382.0 \\
\hline G14200 & 2458 & 2456 & 2452.1 & 985 & 2456 & 2452.4 & 361 & 2418 & 1121.1 & 2416 & 351.0 \\
\hline G14300 & 2442 & 2440 & 2432.9 & 491 & 2438 & 2435.5 & 551 & 2410 & 1215.8 & 2408 & 377.0 \\
\hline G14400 & 2450 & 2446 & 2440.2 & 1739 & 2448 & 2440.0 & 1036 & 2422 & 1237.2 & 2414 & 356.0 \\
\hline G14500 & 2446 & 2446 & 2437.9 & 877 & 2444 & 2438.7 & 1193 & 2416 & 1122.5 & 2406 & 388.0 \\
\hline G14600 & 2450 & 2448 & 2441.2 & 1163 & 2448 & 2442.3 & 884 & 2424 & 1213.9 & 2412 & 331.0 \\
\hline G14700 & 2444 & 2440 & 2431.5 & 1829 & 2440 & 2435.0 & 1384 & 2404 & 1230.6 & 2410 & 381.0 \\
\hline G14800 & 2448 & 2442 & 2436.9 & 1725 & 2444 & 2438.9 & 1055 & 2416 & 1132.0 & 2418 & 332.0 \\
\hline G14900 & 2426 & 2422 & 2414.7 & 1605 & 2422 & 2417.3 & 1185 & 2412 & 1213.9 & 2388 & 333.0 \\
\hline G141000 & 2458 & 2452 & 2445.8 & 2097 & 2454 & 2448.8 & 1345 & 2430 & 1125.8 & 2420 & 391.0 \\
\hline Average & & & & 473.2 & & & 377.5 & & 537.3 & & 163.2 \\
\hline Better & & 0 & & & 0 & & & 0 & & 0 & \\
\hline Equal & & 21 & & & 20 & & & 10 & & 10 & \\
\hline Worse & & 9 & & & 10 & & & 20 & & 20 & \\
\hline
\end{tabular}

Table 9

Computational Results on MaxCut Instances of Set3

\begin{tabular}{|c|c|c|c|c|c|c|c|c|c|c|c|}
\hline \multirow[b]{2}{*}{ Instance } & \multirow[b]{2}{*}{$f_{\text {prev }}$} & \multicolumn{3}{|c|}{ PR1 } & \multicolumn{3}{|c|}{ PR2 } & \multicolumn{2}{|c|}{ SS [29] } & \multicolumn{2}{|c|}{ CirCut [8] } \\
\hline & & $f_{\text {best }}$ & $f_{\text {avr }}$ & $\overline{\text { time }}$ & $f_{\text {best }}$ & $f_{\text {avr }}$ & $\overline{\text { time }}$ & $f_{\text {best }}$ & time & $f_{\text {best }}$ & time \\
\hline g3-15 & 283206561 & 279830931 & 277345801.1 & 3000 & 276903146 & 273564256.6 & 1272 & 281029888 & 1023 & 268519648 & 788 \\
\hline g3-8 & 41684814 & 41684814 & 41508934.7 & 292 & 41684814 & 41521529.9 & 258 & 40314704 & 66 & 41684814 & 54 \\
\hline $\begin{array}{l}\text { pm3- } \\
15-50\end{array}$ & 3000 & 3010 & 3006.6 & 1602 & 3014 & 3007.3 & 1890 & 2964 & 333 & 2895 & 427 \\
\hline $\begin{array}{c}\mathrm{pm} 3-8- \\
50\end{array}$ & 458 & 458 & 458.0 & 2 & 458 & 458.0 & 2 & 442 & 49 & 454 & 39 \\
\hline Average & & & & 1224.0 & & & 855.5 & & 367.7 & & 326.9 \\
\hline Better & & 1 & & & 1 & & & 0 & & 0 & \\
\hline Equal & & 2 & & & 2 & & & 0 & & 1 & \\
\hline Worse & & 1 & & & 1 & & & 4 & & 3 & \\
\hline
\end{tabular}


Table 10

Computational Results on MaxCut with longer CPU time

\begin{tabular}{ccccccccc}
\hline Instance & $f_{\text {best }}$ & time & Instance & $f_{\text {best }}$ & time & Instance & $f_{\text {best }}$ & time \\
\hline G25 & 13340 & 3539 & G27 & 3341 & 3040 & G28 & 3298 & 17482 \\
G30 & 3413 & 4795 & G31 & 3310 & 10801 & G37 & 7686 & 3903 \\
G38 & 7688 & 17230 & G39 & 2408 & 3087 & G40 & 2400 & 11947 \\
G41 & 2405 & 945 & G42 & 2481 & 5580 & G55 & 10274 & 31764 \\
G56 & 3993 & 11727 & G57 & 3484 & 4968 & G58 & 19225 & 20499 \\
G59 & 6039 & 28790 & G60 & 14131 & 62466 & G61 & 5748 & 29056 \\
G62 & 4854 & 59568 & G63 & 26941 & 45136 & G64 & 8693 & 66851 \\
G65 & 5544 & 94934 & G66 & 6340 & 74375 & G67 & 6928 & 114438 \\
G70 & 9529 & 135572 & G72 & 6978 & 141167 & G14100 & 2446 & 2105 \\
G14200 & 2458 & 1657 & G14600 & 2450 & 1476 & G14700 & 2442 & 2824 \\
G14800 & 2446 & 3543 & G14900 & 2426 & 7165 & G141000 & 2458 & 8929 \\
\hline
\end{tabular}

g3-8 with CPU time 292, 258 and 54 seconds, respectively. However, both PR algorithms perform slightly worse than SS on instance g3-15.

To verify whether the proposed PR algorithms are able to further improve the results by allowing longer computational time, we re-ran PR1 and PR2 on the MaxCut instances using 10 times longer time than before, as shown in Table 10. Surprisingly, both PR1 and PR2 can further improve its best results on a total of 33 instances. Although we only show the better results without differentiating whether they come from PR1 or PR2, we find that PR1 and PR2 obtain the same results on 7 instances of set 2, while better results come from PR2 for the 25 instances of set 1 (except the instance G31).

\subsection{Additional Comparisons}

In order to further compare the proposed path relinking algorithms and the HMA algorithm in [27], we apply the time-to-target (TTT) analysis to show the empirical probability distribution of the needed time to attain a given target value [1]. For this experiment, we also include a multistart tabu search algorithm (MSTS) which is the tabu search procedure used in the path relinking algorithms reinforced with a random restart procedure.

We carry out the TTT experiment on a random UBQP instance (p5000.5) and a structured MaxCut instance (G25) with the PR1, PR2, HMA and MSTS algorithms. We perform 200 independent runs for each algorithm and each graph and record the time needed to attain an objective value at least as good as a given target value for each run. Then we sort the recorded times in an increasing order so that $t_{i}$ represents the $i^{\text {th }}$ lowest time and a probability $p_{i}=(i-1 / 2) / 200$ is associated to each time $t_{i}$. Finally, the points $\left(t_{i}, p_{i}\right)$ are plotted. Figure 1 shows the results of the TTT experiment for PR1, PR2, 
HMA and MSTS on the two tested instance p5000.5 (Left) and G25 (Right).
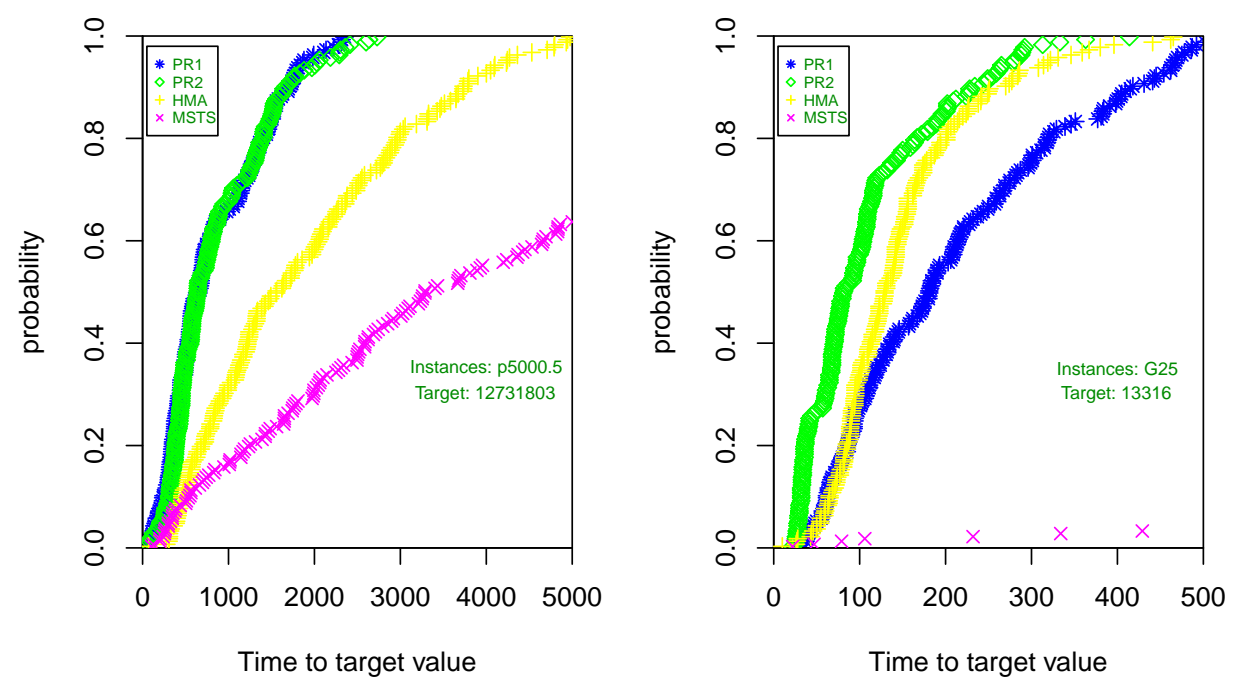

Fig. 1. Empirical probability distribution for the time to achieve a target value

From the left part of Figure 1, we first observe that for the first 400 seconds, PR1, PR2, HMA and MSTS almost perform the same with a low probability of $17 \%$ to reach the target value. Afterwards, PR1 and PR2 are obviously superior to HMA and MSTS. Specifically, at the moment of 2000 seconds, both PR1 and PR2 reach the target value with a probability of $100 \%$ against a probability of $60 \%$ for HMA and a probability of $38 \%$ for MSTS.

From the right part of Figure 1, we notice that MSTS performs much worse than the other algorithms with a probability less than $5 \%$ to reach the target value during the overall time span of 500 seconds while PR1, PR2 and HMA only need 50 seconds to yield an equal or a better performance. In addition, after 50 seconds PR2 always has a higher probability to achieve the target value than HMA and PR1. However, HMA is superior to PR1 from the moment of 100 seconds, which reverses the observation on instance p5000.5 where HMA is generally inferior to PR1. Therefore, this experiment shows that the path relinking procedure, as one of the important components of the proposed PR1 and PR2 algorithms, does play a key role for the good performance of our algorithms, especially in comparison with the MSTS algorithm.

\section{Discussion}

In the previous section, we showed that the proposed path relinking algorithms are able to achieve very competitive results on the UBQP and MaxCut 
benchmark instances. In this section, we discuss the results obtained on two other well-known combinatorial problems: set packing and graph $k$-coloring.

For the set packing problem, we first recast the problem into the UBQP model as shown in [2]. This experiment is based on a set of 16 large random benchmark instances with up to 2000 variables used in $[2,11]$. The experimental results (within a time limit of 30 minutes) show that our path relinking algorithms can match the best known results on 10 of the 16 instances. Remarkably, PR2 is able to improve the best known results ever reported in the literature for 2 instances. This performance can be considered to be very competitive in comparison with the state of the art methods like the GRASP algorithm of [11] which is specially designed for the set packing problem.

For the graph $k$-coloring problem, we recast the problem to the UBQP model according to the transformation shown in [24]. For each graph, we set $k$ to be equal to the smallest known number ever reported in the literature and ran PR2 to check whether PR2 can find a feasible coloring. On the one hand, for the 21 small graph instances considered in [24] with up to 450 vertices and 1000 edges, PR2 can find a feasible coloring for each tested instance. On the other hand, tests on 20 challenging DIMACS graphs indicate that it is very difficult for PR2 to find feasible colorings with $k$ set to be the smallest color number reported in the literature. Indeed, PR2 only found the feasible coloring on 2 out of 20 instances. This experiment indicates that though our path relinking algorithms are able to find good approximate solutions for the $k$-coloring problem, they can not compete with the current best coloring algorithms.

\section{Conclusion}

In this paper, we proposed two effective path relinking algorithms for the unconstrained binary quadratic programming problem. The proposed algorithms are composed of a reference set initialization method, an improvement method by tabu search, a reference set update method, a relinking method and a path solution selection method. The proposed algorithms differ from each other mainly on the way they generate the path, one employing a greedy strategy (PR1) and the other employing a random strategy (PR2). The experiments suggest that PR1 is more appropriate for random instances while PR2 is preferable for structured instances.

Computational experiments on five sets of 134 well-known random and structured benchmark instances have demonstrated that both algorithms are capable of attaining highly competitive results in comparison with the previous best-known results from the literature. In particular, for three sets of bench- 
marks with a total of 103 instances derived from the MaxCut problem, our algorithms can improve the previous best known results for almost 40 percent of these instances whose optimum solutions are still unknown. We also indicated that the path relinking algorithms perform quite well on 16 large set packing benchmark instances, but their performance on $k$-coloring is more moderate. It would be interesting to verify the performance of the proposed algorithms in solving other combinational problems that can be reformulated into the UBQP model.

There are several issues for future consideration. First, more elaborate methods can be used to better manage the reference set by considering both the the quality of solution and its distance to the previously found solutions, given the fact that a good diversity of the reference set is important for the path generation. Second, it would be interesting to verify if selecting more than one solution from a path for improvement is a good strategy. Third, by replacing the basic tabu search based improvement method with a more advanced tabu search method, still better outcomes could be expected.

\section{Acknowledgement}

We would like to thank the anonymous referees for their helpful comments and suggestions. The work is partially supported by the regional RaDaPop (2009-2013) and LigeRo projects (2009-2013).

\section{References}

[1] Aiex RM, Resende MGC, Ribeiro CC (2007) TTT plots: a perl program to create time-to-target plots. Optimization Letters 1: 355-366

[2] Alidaee B, Kochenberger GA, Lewisa K, Lewisc M, Wang HB (2008) A new approach for modeling and solving set packing problems. European Journal of Operational Research 186(2):504-512

[3] Beasley JE (1996) Obtaining test problems via internet. Journal of Global Optimization 8:429-433

[4] Beasley JE (1998) Heuristic algorithms for the unconstrained binary quadratic programming problem. Working Paper, The Management School, Imperial College, London, England

[5] Borgulya I (2005) An evolutionary algorithm for the binary quadratic problems. Advances in Soft Computing 2:3-6 
[6] Boros E, Hammer PL, Sun R, Tavares G (2008) A max-flow approach to improved lower bounds for quadratic 0-1 minimization. Discrete Optimization 5(2):501-529

[7] Boros E, Hammer PL, Tavares G (2007) Local search heuristics for Quadratic Unconstrained Binary Optimization (QUBO). Journal of Heuristics 13:99-132

[8] Burer S, Monteiro RDC, Zhang Y (2001) Rank-two relaxation heuristics for max-cut and other binary quadratic programs. SIAM Journal on Optimization $12: 503-521$

[9] Chardaire P, Sutter A (1994) A decomposition method for quadratic zero-one programming. Management Science 41(4):704-712

[10] Choi C, Ye Y (2000) Solving sparse semidefinite programs using the dual scaling algorithm with an iterative solver. Working paper, Department of Management Sciences, The University of Iowa.

[11] Delorme X, Gandibleau X, Rodriques J (2004) GRASP for set packing. European Journal of Operational Research 153:564-580

[12] Festa P, Pardalos PM, Resende MGC, Ribeiro CC (2002) Randomized heuristics for the max-cut problem. Optimization Methods and Software 7:1033-1058

[13] Glover F, Laguna M (1997) Tabu search. Kluwer Academic Publishers, Boston

[14] Glover F, Kochenberger GA, Alidaee B (1998) Adaptive memory tabu search for binary quadratic programs. Management Science 44:336-345

[15] Glover F, Laguna M, Marti R (2000) Fundamentals of scatter search and pathrelinking. Control and Cybernetics 39:654-684

[16] Glover F, Laguna M, Marti R (2003) Scatter search and path relinking: advances and applications. Handbook of Metaheuristics 57:1-35

[17] Glover F, Laguna M, Marti R (2004) Scatter Search and Path Relinking: Foundations and Advanced Designs, New Optimization Technologies in Engineering, eds. G. C. Onwubolu and B. V. Babu, Studies in Fuzziness and Soft Computing, 141:87-100

[18] Glover F, Hao JK (2010). Efficient Evaluation for Solving 0-1 Unconstrained Quadratic Optimization Problems. International Journal of Metaheuristics 1(1):310

[19] Glover F, Lü Z, Hao JK (2010) Diversification-driven tabu search for unconstrained binary quadratic problems. 4OR: A Quarterly Journal of Operations Research 8(3):239-253

[20] Harary F (1953) On the notion of balanced of a signed graph. Michigan Mathematical Journal 2:143-146

[21] Helmberg C, Rendl F (1998) Solving quadratic (0,1)-problem by semidefinite programs and cutting planes. Mathematical Programming 82:388-399 
[22] Katayama K, Narihisa H (2001) Performance of simulated annealing-based heuristic for the unconstrained binary quadratic programming problem. European Journal of Operational Research 134:103-119

[23] Kochenberger GA, Glover F, Alidaee B, Rego C (2004) A unified modeling and solution framework for combinatorial optimization problems. OR Spectrum $26: 237-250$

[24] Kochenberger GA, Glover F, Alidaee B, Rego C (2005) An unconstrained quadratic binary programming approach to the vertex coloring problem. Annals OR 139(1): 229-241

[25] Krarup J, Pruzan A (1978) Computer aided layout design. Mathematical Programming Study 9:75-84

[26] Lodi A, Allemand K, Liebling TM (1999) An evolutionary heuristic for quadratic 0-1 programming. European Journal of Operational Research $119(3): 662-670$

[27] Lü Z, Glover F, Hao JK (2010) A hybrid metaheuristic approach to solving the UBQP problem. European Journal of Operational Research 207(3):1254-1262

[28] McBride RD, Yormark JS (1980) An implicit enumeration algorithm for quadratic integer programming. Management Science 26:282-296

[29] Marti R, Duarte A, Laguna M (2009) Advanced scatter search for the max-cut problem. INFORMS Journal on Computing 21(1):26-38

[30] Merz P, Freisleben B (1999) Genetic algorithms for binary quadratic programming. In: Proceedings of the Genetic and Evolutionary Computation Conference (GECCO99), Morgan Kaufmann, pp 417-424

[31] Merz P, Katayama K (2004) Memetic algorithms for the unconstrained binary quadratic programming problem. BioSystems 78:99-118

[32] Palubeckis G (2004) Multistart tabu search strategies for the unconstrained binary quadratic optimization problem. Annals of Operations Research 131:259282

[33] Palubeckis G (2004) Application of multistart tabu search to the MaxCut problem. Information Technology and Control 2(31):29-35

[34] Palubeckis G (2006) Iterated tabu search for the unconstrained binary quadratic optimization problem. Informatica 17(2):279-296

[35] Pardalos P, Rodgers GP (1990) Computational aspects of a branch and bound algorithm for quadratic zero-one programming. Computing 45:131-144

[36] Shylo VP, Shylo OV (2010) Solving the maxcut problem by the global equilibrium search. Cybernetics and Systems Analysis 46(5):744-754

[37] Wang Y, Lü Z, Glover F, Hao JK (2011) Backbone guided Tabu Search for solving the UBQP problem. Journal of Heuristics (DOI: 10.1007/s10732-011-91644) 
[38] Wang Y, Lü Z, Glover F, Hao JK (2011) Effective variable fixing and scoring strategies for binary quadratic programming. P. Merz, J.K. Hao (Eds): EvoCOP 2011, Lecture Notes in Computer Science 6622: 72-83. 